\section{Uso de anti-hipertensivos e antidiabéticos por idosos: inquérito em Belo Horizonte, Minas Gerais, Brasil}

\author{
Use of anti-hypertensive and anti-diabetic drugs \\ by the elderly: a survey in Belo Horizonte, \\ Minas Gerais State, Brazil
}

\author{
1 Universidade Federal de \\ Minas Gerais, Belo Horizonte, \\ Brasil. \\ 2 Prefeitura Municipal \\ de Belo Horizonte, Belo \\ Horizonte, Brasil. \\ 3 Escola Nacional de Saúde \\ Pública Sergio Arouca, \\ Fundação Oswaldo Cruz, \\ Rio de Janeiro, Brasil. \\ Correspondência \\ M. F. Gontijo \\ Universidade Federal de Minas \\ Gerais. \\ Rua Castelo Moura 16, \\ Belo Horizonte, $M G$ \\ 31330-210, Brasil. \\ monicagontijo@hotmail.com
}

\section{Abstract}

Concern over the harmful effects of drug use by the elderly has motivated studies aimed at identifying problems in such utilization. This was a household survey with retirees aged $\geq 60$ years living in Belo Horizonte, Minas Gerais State, Brazil, in 2003, who reported having a diagnosis of diabetes and/or hypertension. Quality of anti-hypertensive and anti-diabetic medication was measured by redundancy, combinations of drugs, and inappropriate drugs. Among 283 elderly patients (89\%) with self-reported hypertension and use of anti-hypertensive pharmacotherapy, 68.2\% were using diuretics and $37.8 \%$ ACE inhibitors. Among the 22 (64.7\%) self-reported diabetic patients under pharmacotherapy, 45.5\% were using insulin and $77.3 \%$ oral anti-diabetic agents. Among the 89 self-reported diabetic and hypertensive patients, 80 (90\%) were using anti-hypertensive drugs and 51 (57.3\%) anti-diabetic agents. The study revealed the use of dose combinations, redundant use, and inappropriate medicines, thus indicating the need to monitor treatment protocols and improve healthcare for elderly patients.

Aging; Pharmacoepidemiology; Drug Utilization

\author{
Mônica de Fátima Gontijo 1,2 \\ Andréia Queiroz Ribeiro 1 \\ Carlos Henrique Klein 3 \\ Suely Rozenfeld 3 \\ Francisco de Assis Acurcio ${ }^{1}$
}

\section{Introdução}

Os idosos compõem o grupo etário mais medicalizado na sociedade 1 . O aumento da prevalência de doenças crônico-degenerativas, entre elas hipertensão arterial e diabetes mellitus 2, tem contribuído para o elevado consumo de medicamentos nessa faixa etária. Hipertensão e diabetes são reconhecidos fatores de risco para as doenças cardiovasculares, que configuram importante causa de óbito entre a população idosa 2,3,4,5,6 e, portanto, devem ser tratadas adequadamente. Nesse contexto, são consideradas doenças cujo enfrentamento é prioritário e para as quais existem alternativas terapêuticas capazes de promover seu controle e melhorar a qualidade de vida de seus portadores 3,4 .

Entretanto, a terapia farmacológica para esse grupo etário requer cuidados especiais. A preocupação com os efeitos prejudiciais do uso de medicamentos por idosos tem aumentado e motivado estudos no Brasil, identificando prescrições com associações não justificadas, interações medicamentosas, redundância e uso de medicamentos sem valor terapêutico, o que pode contribuir para o aparecimento de efeitos tóxicos e reações adversas graves 1,7 .

Este trabalho teve por objetivo descrever e avaliar a qualidade de uso dos medicamentos anti-hipertensivos e antidiabéticos utilizados por amostra representativa da população idosa aposentada pelo Instituto Nacional do Seguro 
Social (INSS), residente em Belo Horizonte, Minas Gerais, Brasil, além de examinar aspectos relacionados ao acesso a esses medicamentos.

\section{Materiais e métodos}

Este estudo integra o projeto Perfil de Utilização de Medicamentos por Aposentados Brasileiros, um estudo multicêntrico cuja metodologia e resultados de cobertura estão descritos detalhadamente em Acurcio et al. 8, e foi financiado pela Fundação Nacional de Saúde, Ministério da Saúde (convênio no. 796/2002).

Os dados analisados referem-se ao inquérito domiciliar entre aposentados e pensionistas cadastrados no Ministério da Previdência e Assistência Social, com 60 anos ou mais, residentes em Belo Horizonte, selecionados por amostragem aleatória simples. Foram sorteados 800 indivíduos. Considerando-se um nível de 95\% de confiança, taxas de respostas de $75 \%$ para as entrevistas domiciliares e supondo-se ausência de diferenças relevantes entre respondentes e não respondentes, os 600 entrevistados estimados permitiriam obter erros amostrais entre $4 \%$ e $0,8 \%$ e, para prevalências, entre $50 \%$ e $1 \%$, respectivamente. Devido a perdas inevitáveis, como falecimento, mudança de endereço, foi necessário sorteio adicional de 81 indivíduos ${ }^{8}$. Foram entrevistados 667 indivíduos.

Um questionário estruturado, padronizado e testado foi utilizado para a coleta de dados, com perguntas sobre variáveis sociodemográficas, estado de saúde, uso de serviços e uso de medicamentos nos últimos 15 dias.

Duas perguntas nortearam o agrupamento dos idosos em hipertensos, diabéticos e diabéticos hipertensos, a saber: "Alguma vez na vida, um médico ou outro profissional disse que o senhor(a) tem ou teve pressão alta?" e "Alguma vez na vida, um médico ou outro profissional disse que o senhor(a) tem ou teve diabetes?". Constituíram o grupo de hipertensos os idosos que responderam sim exclusivamente à primeira pergunta; diabéticos aqueles que responderam sim exclusivamente à segunda pergunta; e diabéticos e hipertensos aqueles que responderam sim às duas perguntas simultaneamente.

Foi verificada a prevalência do uso de medicamentos nos 15 dias anteriores à entrevista. As informações sobre os medicamentos foram obtidas a partir da declaração de uso pelos participantes, acompanhada da apresentação das embalagens, bulas ou receitas dos remédios utilizados, independente de prescrição médica.

Aspectos relacionados ao acesso aos medicamentos foram investigados por meio da pergun- ta: "Nos últimos 15 dias, o(a) senhor(a) deixou de usar algum remédio que deveria estar usando?". O motivo de não utilização foi explorado para cada medicamento com resposta afirmativa a essa questão.

Os nomes comerciais foram relacionados aos princípios ativos por meio do Dicionário de Especialidades Farmacêuticas (DEF) 9, e esses foram agrupados de acordo com a classificação ATC - Anatomical Therapeutic Chemical Classification System 10, considerando-se os seguintes níveis: "C" - medicamentos cardiovasculares e "A10" - medicamentos usados no diabetes.

Os medicamentos anti-hipertensivos foram classificados em diuréticos, inibidores da enzima conversora da angiotensina (IECA), bloqueadores de canal de cálcio (BCC), $\beta$-bloqueadores adrenérgicos, antagonistas do receptor da angiotensina (ARA), vasodilatadores periféricos e outros anti-hipertensivos. Os antidiabéticos foram classificados em insulinas e antidiabéticos orais.

A qualidade do uso dos anti-hipertensivos e antidiabéticos foi avaliada com base nos indicadores: presença de redundância farmacológica, uso de associações medicamentosas 1, utilização de fármacos considerados inapropriados para idosos 11 .

Foi considerada redundância a utilização de mais de um princípio ativo de uma mesma classe terapêutica ${ }^{1}$. Associação medicamentosa foi considerada como a combinação de dois ou mais princípios ativos em um mesmo produto comercial.

Foram considerados inadequados para os idosos, de acordo com os critérios de Fick et al. 11, os anti-hipertensivos de baixo índice terapêutico como a metildopa e a clonidina e os antidiabéticos de ação prolongada como a clorpropamida.

As unidades de análise foram os indivíduos e os princípios ativos. Foram estimadas as prevalências de uso de medicamentos e o número médio de princípios ativos consumidos por cada grupo, com seus respectivos desvios-padrão (DP). Foi realizada a distribuição de frequências das classes farmacológicas e de princípios ativos anti-hipertensivos e antidiabéticos utilizados pelos idosos. Também foi descrita a frequência da não utilização de medicamentos que deveriam estar sendo utilizados e os motivos do não uso.

Os softwares utilizados para a organização e análise dos dados foram o Paradox 4.5 (Borland International, Scotts Valley, Estados Unidos), o Excel 2003 (Microsoft Corp., Estados Unidos) e o SPSS 12.0 (SPSS Inc., Chicago, Estados Unidos).

O projeto foi avaliado e aprovado pelo Comitê de Ética em Pesquisa da Universidade Federal de Minas Gerais (processo ETIC 055/03), em cumprimento à Declaração de Helsinki. Todos os 
participantes assinaram o Termo de Consentimento Livre e Esclarecido.

\section{Resultados}

Entre os 667 idosos entrevistados, 89 (13,4\%) se autodeclararam ter diagnóstico de diabetes e hipertensão simultaneamente; 319 (47,8\%) tinham diagnóstico de hipertensão; e 34 (5,1\%), de diabetes.

A idade média dos 319 autodeclarados hipertensos era de 72,4 anos (DP = 7,2 anos); e, entre eles, $65,5 \%$ eram do sexo feminino; e $81,8 \%$ recebiam, como benefício do INSS, menos de três salários mínimos (média = 1,54 SM e DP = 1,4 SM).

Os 34 autodeclarados diabéticos tinham idade média de 72,5 anos ( $D P=8,1$ ); 47,1\% eram mulheres; e $76,5 \%$ recebiam benefício do INSS inferior a três salários mínimos (média = 1,96 SM e DP = 1,7 SM).

Os 89 autodeclarados diabéticos hipertensos tinham idade média de 72,5 anos (DP = 8,2); $79,8 \%$ eram do sexo feminino; e $91 \%$ recebiam menos de três salários mínimos como benefício do INSS (média = 1,16 SM e DP = 1,3 SM).

\section{Uso de medicamentos}

Observou-se um consumo médio de 5,7 (DP = 3,6) medicamentos entre os hipertensos, 4,8 $(\mathrm{DP}=4,5)$ entre os diabéticos e $6,2(\mathrm{DP}=3,7)$ entre os diabéticos hipertensos. As prevalências de uso de, pelo menos, um medicamento de qualquer classe farmacológica, na última quinzena anterior à entrevista, foram de 96,5\%, 88,2\% e $97,8 \%$ entre os hipertensos, os diabéticos e entre os diabéticos hipertensos, respectivamente. Foram encontrados, em cada grupo, usuários de até 19, 23 e 18 produtos, respectivamente.

\section{- Entre os hipertensos}

A prevalência do uso de anti-hipertensivo foi de $88,7 \%$ ( $n=283$ ). A associação de dois anti-hipertensivos foi observada em cerca de $1 / 3$ dos hipertensos, seguida pela monoterapia (Tabela 1).

A subamostra de idosos hipertensos utilizou 811 princípios ativos com ação cardiovascular. Desse total, 705 (86,9\%) eram anti-hipertensivos. Entre os hipertensos em uso de anti-hipertensi$\operatorname{vos}(n=283)$, a classe de fármacos mais frequentemente empregada para controle da doença era a de diuréticos $(68,2 \%)$, especialmente a hidroclorotiazida. A segunda classe mais utilizada foi a de IECA (38\%), sobressaindo-se o enalapril e captopril. A nifedipina foi o princípio ativo mais utilizado entre os BCC, e o propranolol, entre os $\beta$-bloqueadores. A metildopa se destacou em seu subgrupo farmacológico (Tabela 2).

\section{- Entre os diabéticos}

A prevalência de uso de antidiabéticos foi de $64,7 \%$ ( $n=22$ ). O uso de monoterapia foi observado em $32,4 \%(n=11)$ dos diabéticos (Tabela 1$)$.

A subamostra de idosos diabéticos usou 117 princípios ativos antidiabéticos, sendo 24,8\% insulinas e $75,2 \%$ antidiabéticos orais. Entre os diabéticos usuários de farmacoterapia $(n=22)$, o uso de antidiabéticos orais era muito frequente, principalmente a metformina $(64,7 \%)$ e a glibenclamida (52,9\%) (Tabela 2$)$.

\section{- Entre os diabéticos e hipertensos}

Entre os 89 diabéticos hipertensos, a prevalência de uso de anti-hipertensivos foi de $89,9 \%$ ( $n=$ 80) e de antidiabéticos, de $57,3 \%(n=51)$. Nesse grupo, $38,2 \%, 32,6 \%$ e $20,1 \%$ utilizavam monoterapia, dois ou três ou mais anti-hipertensivos, respectivamente, e $33,7 \%, 19,1 \%$ e $4,5 \%$ utilizavam monoterapia, dois ou três ou mais antidiabéticos, respectivamente (Tabela 1).

Entre os 80 diabéticos e hipertensos utilizando farmacoterapia, as classes anti-hipertensivas mais utilizadas foram IECA $(51,3 \%)$, diuréticos $(42,5 \%)$, BCC (38,8\%) e $\beta$-bloqueadores $(23,8 \%)$. Os princípios ativos mais utilizados foram o enalapril, a hidroclorotiazida e a nifedipina. A glibenclamida foi o antidiabético oral mais utilizado (Tabela 2).

\section{Não utilização de medicamentos que deveriam estar sendo usados}

Entre os 363 idosos em uso de farmacoterapia anti-hipertensiva (283 hipertensos e 80 diabéticos hipertensos), 90 (24,8\%) deixaram de usar algum medicamento, sendo que $40(11,1 \%)$ deles deixaram de utilizar algum anti-hipertensivo. Entre os 73 idosos em uso de farmacoterapia antidiabética, 8 (10,9\%) deixaram de tomar algum antidiabético (dados não apresentados em tabela). Entre os principais motivos do não uso desses medicamentos, estão a falta de recursos financeiros para adquiri-los (24\%), a falta do produto na farmácia do SUS $(19,5 \%)$ e o esquecimento $(17,5 \%)$ (Tabela 3$)$.

\section{Associações medicamentosas}

Entre os 319 hipertensos, 283 (88,7\%) faziam uso de medicamentos, sendo que $183(57,4 \%)$ utilizavam dois ou mais anti-hipertensivos, e um deles 
Tabela 1

Prevalência do uso de anti-hipertensivos, antidiabéticos e outros princípios ativos de acordo com o grupo de idosos e número de princípios ativos utilizados. Belo Horizonte, Minas Gerais, Brasil, 2003.

\begin{tabular}{|c|c|c|c|c|c|c|}
\hline \multirow[t]{2}{*}{ Variáveis } & \multicolumn{2}{|c|}{ Hipertensos } & \multicolumn{2}{|c|}{ Diabéticos } & \multicolumn{2}{|c|}{ Hipertensos e diabéticos } \\
\hline & $\mathrm{n}$ & Prevalência (\%) & $\mathrm{n}$ & Prevalência (\%) & $\mathrm{n}$ & Prevalência (\%) \\
\hline \multicolumn{7}{|c|}{ Número de anti-hipertensivos } \\
\hline 0 & 36 & 11,3 & - & - & 9 & 10,1 \\
\hline 1 & 100 & 31,3 & - & - & 34 & 38,2 \\
\hline 2 & 110 & 34,5 & - & - & 29 & 32,6 \\
\hline 3 & 58 & 18,2 & - & - & 11 & 12,4 \\
\hline 4 & 14 & 4,4 & - & - & 5 & 6,7 \\
\hline $5 \mathrm{ou}+$ & 1 & 0,3 & - & - & 1 & 1,0 \\
\hline Total & 319 & 100,0 & - & - & 89 & 100,0 \\
\hline \multicolumn{7}{|c|}{ Número de antidiabéticos } \\
\hline 0 & - & - & 12 & 35,3 & 38 & 42,7 \\
\hline 1 & - & - & 11 & 32,4 & 30 & 33,7 \\
\hline 2 & - & - & 8 & 23,5 & 17 & 19,1 \\
\hline 3 & - & - & 2 & 5,9 & 3 & 3,4 \\
\hline 4 & - & - & 1 & 2,9 & 1 & 1,1 \\
\hline $5 \mathrm{ou}+$ & - & - & 0 & 0,0 & 0 & 0,0 \\
\hline Total & - & - & 34 & 100,0 & 89 & 100,0 \\
\hline \multicolumn{7}{|c|}{$\begin{array}{l}\text { Número de anti-hipertensivos + } \\
\text { antidiabéticos }\end{array}$} \\
\hline 0 & - & - & - & - & 6 & 6,7 \\
\hline 1 & - & - & - & - & 15 & 16,9 \\
\hline 2 & - & - & - & - & 26 & 29,2 \\
\hline 3 & - & - & - & - & 22 & 24,7 \\
\hline 4 & - & - & - & - & 13 & 14,6 \\
\hline $5 \mathrm{ou}+$ & - & - & - & - & 7 & 7,9 \\
\hline Total & - & - & - & - & 89 & 100,0 \\
\hline
\end{tabular}

Tabela 2

Prevalência do uso dos diversos subgrupos terapêuticos e princípios ativos anti-hipertensivos e antidiabéticos de acordo com o grupo de idosos (hipertensos e/ou diabéticos) usuários desses produtos. Belo Horizonte, Minas Gerais, Brasil, 2003.

\begin{tabular}{lcccccc}
\hline $\begin{array}{l}\text { Subgrupo terapêutico e subgrupo } \\
\text { farmacológico/Princípios ativos }\end{array}$ & \multicolumn{2}{c}{ Hipertensos } & \multicolumn{2}{c}{ Diabéticos } & \multicolumn{2}{c}{$\begin{array}{c}\text { Hipertensos e } \\
\text { diabéticos }\end{array}$} \\
& $\mathbf{n}$ & $\%$ & $\mathbf{n}$ & $\%$ & $\mathbf{n}$ & $\%$ \\
\hline Diuréticos & & & & & & \\
Hidroclorotiazida * & 131 & 67,9 & - & - & 22 & 64,7 \\
Furosemida & 25 & 13,0 & - & - & 7 & 20,6 \\
Clortalidona & 29 & 15,0 & - & - & 1 & 2,9 \\
Amilorida & 19 & 9,8 & - & - & 1 & 2,9 \\
Outros & 16 & 8,3 & - & - & 8 & 23,5 \\
Total ** & 193 & 68,2 & - & - & 34 & 42,5 \\
\hline
\end{tabular}

(continua) 
Tabela 2 (continuação)

\begin{tabular}{|c|c|c|c|c|c|c|}
\hline \multirow[t]{2}{*}{$\begin{array}{l}\text { Subgrupo terapêutico e subgrupo } \\
\text { farmacológico/Princípios ativos }\end{array}$} & \multicolumn{2}{|c|}{ Hipertensos } & \multicolumn{2}{|c|}{ Diabéticos } & \multicolumn{2}{|c|}{$\begin{array}{c}\text { Hipertensos e } \\
\text { diabéticos }\end{array}$} \\
\hline & $\mathrm{n}$ & $\%$ & $\mathrm{n}$ & $\%$ & $\mathrm{n}$ & $\%$ \\
\hline \multicolumn{7}{|c|}{ Inibidores da enzima conversora da angiotensina } \\
\hline Enalapril & 49 & 45,8 & - & - & 23 & 56,1 \\
\hline Captopril * & 44 & 41,1 & - & - & 17 & 41,5 \\
\hline Outros & 14 & 13,1 & - & - & 1 & 2,4 \\
\hline Total ** & 107 & 37,8 & - & - & 41 & 51,3 \\
\hline \multicolumn{7}{|l|}{ Bloqueadores do canal de cálcio } \\
\hline Nifedipina & 41 & 45,1 & - & - & 18 & 58,1 \\
\hline Anlodipina & 23 & 25,3 & - & - & 5 & 16,1 \\
\hline Diltiazem & 11 & 12,1 & - & - & 4 & 12,9 \\
\hline Outros & 16 & 17,6 & - & - & 4 & 12,9 \\
\hline Total ** & 91 & 32,2 & - & - & 31 & 38,8 \\
\hline \multicolumn{7}{|l|}{$\beta$-bloqueadores } \\
\hline Propranolol * & 57 & 65,5 & - & - & 14 & 73,7 \\
\hline Atenolol & 19 & 21,8 & - & - & 5 & 26,3 \\
\hline Outros & 11 & 12,6 & - & - & 1 & 5,3 \\
\hline Total ** & 87 & 30,7 & - & - & 19 & 23,8 \\
\hline \multicolumn{7}{|c|}{ Antagonistas dos receptores da angiotensina } \\
\hline Losartan & 7 & 38,9 & - & - & 2 & 66,7 \\
\hline Irbesartan & 4 & 22,2 & - & - & 0 & 0,0 \\
\hline Outros & 7 & 38,9 & - & - & 1 & 33,3 \\
\hline Total * & 18 & 6,4 & - & - & 3 & 3,8 \\
\hline \multicolumn{7}{|l|}{ Anti-hipertensivos } \\
\hline Metildopa & 7 & 46,7 & - & - & 3 & 75,0 \\
\hline Hidralazina & 4 & 26,7 & - & - & 0 & 0,0 \\
\hline Outros & 4 & 26,7 & - & - & 1 & 25,0 \\
\hline Total ** & 15 & 5,3 & - & - & 4 & 5,0 \\
\hline \multicolumn{7}{|l|}{ Vasodilatadores periféricos } \\
\hline Pentoxifilina & 1 & 16,7 & - & - & 5 & 83,3 \\
\hline Diidroergocristina & 4 & 66,7 & - & - & 2 & 33,3 \\
\hline Outros & 1 & 16,7 & - & - & 2 & 33,3 \\
\hline Total ** & 6 & 2,1 & - & - & 6 & 11,3 \\
\hline Total geral $\star \star \star ~$ & 283 & 88,7 & - & - & 80 & 89,9 \\
\hline \multicolumn{7}{|l|}{ Medicamentos usados no diabetes } \\
\hline \multicolumn{7}{|l|}{ Antidiabéticos orais } \\
\hline Sulfonilureia: glibenclamida * & - & - & 9 & 52,9 & 26 & 63,4 \\
\hline Biguanidas: metformina * & - & - & 11 & 64,7 & 20 & 48,8 \\
\hline Outros & - & - & 4 & 23,5 & 8 & 19,5 \\
\hline Total ** & - & - & 17 & 77,3 & 41 & 80,4 \\
\hline \multicolumn{7}{|l|}{ Insulinas } \\
\hline Total ** & - & - & 10 & 45,5 & 16 & 31,4 \\
\hline 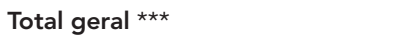 & - & - & 22 & 64,7 & 51 & 57,3 \\
\hline
\end{tabular}

* Princípios ativos pertencentes ao Hiperdia;

** Total de pacientes usando, pelo menos, um dos princípios ativos do subgrupo;

*** Total de indivíduos do grupo que fizeram uso de algum medicamento do grupo anti-hipertensivo ou hipoglicemiante.

Nota: o somatório total dos indivíduos das diversas classes farmacológicas ultrapassa o total de indivíduos do grupo devido à possibilidade de uso de mais de um princípio ativo por idoso. 


\section{Tabela 3}

Motivos apontados entre os grupos de idosos (hipertensos e/ou diabéticos) por deixar de usar algum medicamento anti-hipertensivo e antidiabético. Belo Horizonte, Minas Gerais, Brasil, 2003.

\begin{tabular}{lcc}
\hline Motivo de não uso dos medicamentos & $\mathbf{n}$ & $\%$ \\
\hline Preço/falta de dinheiro & 11 & 24,0 \\
Faltou no SUS & 9 & 19,5 \\
Faltou no comércio & 1 & 2,1 \\
Passou mal com o remédio & 2 & 4,2 \\
Esqueceu de tomar & 8 & 17,5 \\
Melhorou & 4 & 8,8 \\
Outros motivos & 11 & 23,9 \\
Total & 46 & 100,0 \\
\hline
\end{tabular}

utilizava cinco ou mais (Tabela 1). Dentre os usuários de farmacoterapia, 55 (19,4\%) faziam uso de associações de anti-hipertensivos. As associações mais observadas foram de dois diuréticos $(36,7 \%)$, seguida por diurético + IECA $(16,4 \%)$ e diurético + $\beta$-bloqueador (14,5\%). A utilização de associações foi proporcionalmente maior entre os idosos hipertensos com 80 anos ou mais (dados não apresentados em tabela).

Entre os 34 diabéticos, 11 (32,4\%) utilizavam entre dois e quatro antidiabéticos (Tabela 1). Não foram observadas associações medicamentosas neste grupo.

Entre os 89 diabéticos hipertensos, 80 (89,9\%) faziam uso de anti-hipertensivos, sendo que 46 $(58,3 \%)$ utilizavam dois ou mais (dados não apresentados em tabela). Entre os usuários de farmacoterapia, 3 (3,7\%) utilizavam anti-hipertensivos em associação, sendo observado o uso de dois diuréticos (Tabela 4), tais como diurético + betabloqueador e diurético + BCC. Nesse grupo, $51(57,3 \%)$ utilizavam farmacoterapia hipoglicemiante, sendo que 21 (23,6\%) utilizavam dois ou mais (dados não apresentados em tabela). Entre os usuários dessa farmacoterapia, 2 (3,9\%) utilizavam associações medicamentosas (Tabela 4), tais como metformina + glibenclamida $(4,2 \%)$ e metformina + nateglinida $(4,2 \%)$.

\section{Redundância farmacêutica}

Entre os 363 idosos utilizando anti-hipertensivos, a redundância foi observada em 12,9\% dos entrevistados. Desses idosos, 37 faziam uso redundante de diuréticos; quatro, de IECA; três, de vasodilatadores; e dois deles, de $\beta$-bloqueadores (Tabela 4).
Entre os 73 idosos em uso de antidiabéticos orais, $4,1 \%$ faziam uso de redundância farmacêutica (Tabela 4).

\section{Medicamentos inadequados}

Foi observado o uso de anti-hipertensivos e antidiabéticos inadequados, segundo critérios adotados em, aproximadamente, $2 \%$ da amostra. O uso de anti-hipertensivos considerados inadequados para idosos, como a metildopa e a clonidina, foi observado em 10 e 1 participantes, respectivamente. Dois participantes utilizavam a clorpropamida, hipoglicemiante considerado inadequado para idosos.

\section{Discussão}

Observou-se ampla utilização de medicamentos entre os entrevistados que informaram ter diagnóstico de hipertensão arterial, diabetes mellitus ou as duas condições simultaneamente com o uso de medicamentos redundantes e até mesmo impróprios para esse grupo populacional, sugerindo a necessidade de melhoria contínua na farmacoterapia destinada aos mesmos. Por outro lado, 22,5\% dos idosos deixaram de tomar algum medicamento que deveria estar

Tabela 4

Frequência de redundância * farmacêutica entre os grupos de idosos (hipertensos ou diabéticos) e as principais classes farmacológicas anti-hipertensivas e antidiabéticas utilizadas nessa condição. Belo Horizonte, Minas Gerais, Brasil, 2003.

\begin{tabular}{lcc}
\hline Classe terapêutica & $\mathbf{n}$ & $\%$ \\
\hline Diuréticos & $363 * \star$ & \\
IECA & 37 & 10,1 \\
Vasodilatadores & 4 & 1,2 \\
B-bloqueadores & 3 & 0,9 \\
BCC & 2 & 0,6 \\
Total & 1 & 0,3 \\
& 47 & 12,9 \\
Antidiabéticos & $73 * *$ & 4,1 \\
Total & 3 & 4,1 \\
\hline BC: bloqueador de & 3 & \\
\hline
\end{tabular}

BCC: bloqueador de canal de cálcio; IECA: inibidor enzima conversora da angiotensina.

* Redundância foi considerada a utilização de mais de um princípio ativo de uma mesma classe terapêutica;

** Número de idosos hipertensos e/ou diabéticos em uso de farmacoterapia. 
em uso, sendo a falta de recursos financeiros para adquiri-los a principal justificativa da não utilização. Esses resultados ilustram bem o cenário que se configura pelo dilema "acesso e excesso" no tocante à farmacoterapia nessa população, sendo necessárias ações sistemáticas que visem racionalizar o uso de medicamentos por idosos.

A informação sobre as doenças obtida por meio de declaração dos entrevistados, embora com validade comprovada em outros estudos 1,5 , sugere que as estimativas devam ser observadas com cautela. Pode ter havido subestimação das prevalências pela exclusão daqueles que desconheciam a doença ou que não conseguiram assimilar a informação diagnóstica. Por outro lado, o enunciado da pergunta inclui a possibilidade de diagnóstico por um profissional não qualificado para provê-lo, superestimando a medida. A morbidade autorreferida pode ser ainda influenciada pelo acesso e uso de serviços médicos, sendo moldada, por um lado, pela capacidade de procura e obtenção do cuidado pelo usuário e, de outro, pela organização da atenção que definirá a oferta, a qualidade e a resolubilidade das ações e serviços. Entretanto, diferentes estudos têm demonstrado a utilidade desse tipo de abordagem 2,6,12,13.

A prevalência do uso de anti-hipertensivos, entre os hipertensos, foi elevada $(88,7 \%)$, revelando a importância de se estudar esses produtos, que estão entre os mais consumidos por ido$\operatorname{sos} 7,14,15,16$. Vale destacar a elevada frequência de terapia combinada, o que corrobora a literatura no sentido de que a maioria dos idosos necessita do uso de mais de um anti-hipertensivo, principalmente para o controle adequado da pressão arterial sistólica ${ }^{3}$. As classes anti-hipertensivas mais utilizadas, em todos os grupos estudados, foram os diuréticos, IECA e $\beta$-bloqueadores, provavelmente por serem fornecidos pelo Sistema Único de Saúde (SUS).

Os diuréticos foram os mais prescritos, concordando com as recomendações de diferentes guias, formulários e diretrizes que os consideram a classe anti-hipertensiva mais valiosa para a população idosa 3,17 (Micromedex Health Series. https://www.thomsonhc.com/hcs/li brarian/CS/81329C/PFPUI/tBLDVw321t9WW/ PFActionId/pf.HomePage/ssl/true, acessado em 16/Jun/2009). Tais fármacos são considerados de primeira linha para o tratamento da hipertensão devido à eficácia em reduzir eventos cardiovasculares, à baixa incidência de eventos adversos, à comodidade posológica e ao baixo custo 3,5,17,18,19,20 (Micromedex Health Series. https://www.thomsonhc.com/hcs/librarian/ CS / 81329C/ PFPUI/tBLDVw321t9WW/PFAc
tionId/pf.HomePage/ssl/true, acessado em 16/ Jun/2009).

Verificou-se ampla utilização dos IECA para tratamento da hipertensão entre os diabéticos hipertensos. Esse medicamento tem sido recomendado por reduzir a proteinúria e retardar o declínio da função renal, além de preservar a função ventricular 4,18,19 (Micromedex Health Series. https://www.thomsonhc.com/hcs/librarian/ CS / 81329C/PFPUI/ tBLDVw321 t9WW/PFAc tionId/pf.HomePage/ssl/true, acessado em 16/ Jun/2009). Uma metanálise com mais de 13 mil diabéticos demonstrou que o uso de IECA reduz, em $20 \%$, a mortalidade e, em $55 \%$, a progressão da doença renal 21.

Os betabloqueadores foram utilizados com frequência, estando em acordo com as recomendações do VII Joint National Committee on Prevention, Detection, Evaluation, and Treatment of High Blood Pressure 22, que os considera a escolha inicial, juntamente com os diuréticos, no tratamento da hipertensão, não complicada e, para aqueles pacientes com infarto do miocárdio, sem atividade simpatomimética intrínseca.

Comparando o consumo de cada classe de medicamentos, observam-se algumas diferenças com os resultados de outras publicações 1,5,16. Diferentes perfis de utilização de medicamentos podem refletir diversidades nos estados de saúde das populações, nos modelos de atenção e frequência de utilização dos serviços, além de traços demográficos e culturais relacionados ao consumo de medicamentos 15,16.

É importante destacar que, aproximadamente, um décimo dos pacientes hipertensos e um terço dos diabéticos não relataram uso de fármacos para o seu tratamento. Possíveis explicações para esse fato incluem a opção pelo tratamento não farmacológico, o que é compatível com as recomendações e diretrizes para tratamento do diabetes, que sugere mudanças de hábitos e estilo de vida como forma de controle da doença 4 , recomendação também válida para a hipertensão. Para determinados pacientes, o tratamento não farmacológico pode ser suficiente para obter esse controle. Outro aspecto que pode ter contribuído para a não utilização dos medicamentos refere-se às barreiras existentes ao seu acesso. Ainda que o SUS procure garantir a oferta contínua de medicamentos anti-hipertensivos e antidiabéticos na rede básica de saúde, este estudo identificou pacientes diabéticos e/ou hipertensos que deixaram de utilizar medicamentos indicados. Entre os principais motivos apresentados, encontram-se a falta do produto na farmácia do SUS e dificuldades financeiras para sua aquisição. Essa situação preocupa, pois o adequado controle dessas doenças é fundamental para a 
qualidade de vida dos seus portadores. Problemas de adesão ao tratamento também podem contribuir para explicar parcialmente o não uso de terapia farmacológica entre os participantes. Outra possível explicação está relacionada às limitações inerentes ao diagnóstico autorreferido, que, nem sempre, coincide com aquele obtido por meio de medidas objetivas. A baixa acurácia pode estar relacionada ao desconhecimento das condições de saúde pelo paciente e à incapacidade de lembrar as informações fornecidas pelos seus médicos 12 .

A quantidade de princípios ativos consumida é outro aspecto que deve ser destacado, pois o idoso portador de doenças crônicas também faz uso de outros medicamentos. Os resultados mostram que o número médio de princípios ativos consumidos por diabéticos $(4,8$; $\mathrm{DP}=4,5)$ ou hipertensos $(5,6$; $\mathrm{DP}=3,6)$ é elevado, o que parece confirmar uma prática de utilização de diversas especialidades médicas entre os idosos. Essa média foi maior que a encontrada para o conjunto da população idosa em Belo Horizonte $(4,1) 15$ e no Rio de Janeiro $(3,7)$ 16, o que reforça a importância das doenças crônicas no incremento da utilização de medicamentos. O consumo de medicamentos aumentou quando o participante possuía as duas condições simultaneamente $(6,2 ; \mathrm{DP}=3,71)$. Sabe-se que a presença do diabetes mellitus associado à hipertensão arterial aumenta, em duas vezes, o risco de desenvolver doença cardiovascular 3,4,17,22, sendo a utilização de medicamentos uma das principais medidas para tentar reduzir esse risco.

Neste estudo, foi observado o uso, por alguns pacientes, de até seis diferentes tipos de anti-hipertensivos. Entretanto, esse uso deve ser cauteloso por facilitar a ocorrência de eventos adversos, interações medicamentosas e iatrogenias.

Entre as associações medicamentosas antihipertensivas, foi observada a combinação irracional de $\beta$-bloqueador + IECA. Nesse caso, os $\beta$-bloqueadores causam diminuição da secreção de renina, o que provoca redução dos níveis de angiotensina II, reduzindo o efeito hipotensor dos IECA (Micromedex Health Series. https://www.thomsonhc.com/hcs/librarian/ CS / 81329C/PFPUI/tBLDVw321t9WW/PFAc tionId/pf.HomePage/ssl/true, acessado em 16/ Jun/2009).

Uma limitação do estudo é a abordagem seccional. Talvez as estimativas que mais tenham sofrido impacto do desenho sejam as relacionadas às associações entre fármacos ou as redundâncias, uma vez que a informação sobre uso nos 15 dias que antecederam as entrevistas pode captar a troca de medicamentos no período e não o uso simultâneo. Ainda assim, as estimativas podem ser consideradas válidas, uma vez que dificilmente a troca de marcas justificaria $4,4 \%$ dos hipertensos ou $6,7 \%$ dos diabéticos serem tratados com 4 ou mais produtos.

A maioria dos idosos hipertensos utilizava mais de um princípio ativo anti-hipertensivo. É possível que parte desses indivíduos não tenha aderido adequadamente ao tratamento inicial, por opção ou falta de entendimento da prescrição, ou tenha tido o seu regime posológico modificado ao longo do tempo, em função de aumento da complexidade da condição hipertensiva. Nessa última situação, é possível que tenha havido a necessidade de adição de novos princípios ativos. Corrobora essa hipótese o fato de que, no estudo, entre os hipertensos com 60-79 anos, 15,8\% utilizavam associações anti-hipertensivas. Já entre aqueles com 80 anos e mais, 38,5\% usavam tais associações (dados não mostrados). Essa situação, independente de sua justificativa, favorece o aparecimento de eventos adversos e interações medicamentosas. Por último, vale ressaltar que a adesão terapêutica não foi investigada no presente trabalho, mas é um aspecto fundamental para a efetividade do tratamento e merece especial atenção em estudos futuros.

O uso simultâneo de dois ou mais antidiabéticos foi verificado em, aproximadamente, um terço dos pacientes diabéticos. Segundo a literatura, com os anos ou décadas de evolução do diabetes mellitus, ocorre progressiva redução da capacidade secretória de insulina pelas células $\beta$ pancreáticas, e a monoterapia pode falhar na manutenção do bom controle metabólico 4 (Micromedex Health Series. https://www.thom sonhc.com/hcs/librarian/CS/81329C/PFPUI/ tBLDVw321 t9wW/PFActionId/pf.HomePage/ ssl/true, acessado em 16/Jun/2009).

Observou-se ainda o uso redundante de medicamentos em todos os grupos de anti-hipertensivos utilizados, especialmente de diuréticos. Eventos de redundância envolvendo os BCC foram descritos 1 e interpretados como indício de má qualidade da prescrição médica e ressaltam a importância da avaliação adequada durante a prescrição, visando intervir na qualidade e quantidade de medicamentos consumidos. Também foi demonstrada redundância na condução dos tratamentos antidiabéticos.

O uso de metildopa, anti-hipertensivo contraindicado para idosos 3,11 (Micromedex Health Series. https://www.thomsonhc.com/hcs/ librarian/CS/81329C/PFPUI/tBLDVw321t9WW/ PFActionId/pf.HomePage/ssl/true, acessado em 16/Jun/2009) por causar bradicardia e exacerbar crises depressivas, foi observado em $2,5 \%$ dos entrevistados, semelhante ao encontrado por outros autores 23 . Ainda foi observado o uso de clo- 
nidina, medicamento contraindicado por causar sedação, depressão e hipotensão ortostática 3,11,17 (Micromedex Health Series. https://www.thom sonhc.com/hcs/librarian/CS/81329C/PFPUI/ tBLDVw321t9WW/PFActionId/pf.HomePage/ ssl/true, acessado em 16/Jun/2009). Entre os antidiabéticos, foi observado o uso de clorpropamida, medicamento contraindicado por ter meia-vida longa 4,11 (Micromedex Health Series. https://www.thomsonhc.com/hcs/librarian/ CS / 81329C/ PFPUI/tBLDVw321 t9WW/PFAc tionId/pf.HomePage/ssl/true, acessado em 16/ Jun/2009), podendo prolongar a hipoglicemia. O uso inadequado da clorpropamida em idosos também foi observado em outro estudo deste projeto, realizado no Rio de Janeiro 17.

$\mathrm{O}$ uso de medicamentos em idosos merece atenção, pois, com o avanço da idade, diminui a massa muscular corporal, e ficam comprometidos o metabolismo hepático e a excreção renal. Isso leva à dificuldade de eliminação dos metabólitos e à produção de reações adversas. $\mathrm{O}$ uso de múltiplos medicamentos favorece o aparecimento de eventos adversos e interações medicamentosas nesse grupo etário 1,7,17. Um importante fator preditivo para o uso impróprio de medicamentos é o número total de produtos consumidos, sendo esperado que um quarto dos

\section{Resumo}

A preocupação com efeitos prejudiciais do uso de medicamentos por idosos tem motivado estudos com o objetivo de identificar problemas nessa utilização. Realizou-se um inquérito domiciliar entre aposentados, com idade $\geq 60$ anos, residentes em Belo Horizonte, Minas Gerais, Brasil (2003), que declararam ter diabetes ou hipertensão arterial. A qualidade do uso de medicamentos anti-hipertensivos e antidiabéticos foi avaliada com base em redundância, associações medicamentosas e fármacos inapropriados. Entre os 283 (89\%) idosos autodeclarados hipertensos, em uso de farmacoterapia, 68,2\% utilizavam diuréticos, e 37,8\% utilizavam IECA. Entre os 22 (64,7\%) autodeclarados diabéticos sob farmacoterapia, 45,5\% utilizavam insulina, e 77,3\%, antidiabéticos orais. Entre os 89 autodeclarados diabéticos hipertensos, 80 (90\%) utilizavam anti-hipertensivos, e 51 (57,3\%), antidiabéticos. Observou-se o uso de associações medicamentosas, medicamentos redundantes ou inadequados, o que indica a necessidade de seguimento de protocolos terapêuticos e maior atenção à saúde dos pacientes idosos.

Envelhecimento; Farmacoepidemiologia; Uso de Medicamentos indivíduos que usam três ou mais produtos utilizem, pelo menos, um inadequado ${ }^{1}$.

A elaboração de protocolos terapêuticos, com a padronização de ações que resultem em melhores cuidados e adesão do paciente faz parte do compromisso público da construção do Pacto pela Saúde, assumido pelos gestores do SUS. Esses protocolos devem ser baseados nos princípios constitucionais do SUS, com ênfase nas necessidades de saúde da população, na efetividade do cuidado e na eficiência dos gastos públicos. Um estudo desenvolvido em Gana demonstrou que os gastos do setor farmacêutico poderiam ser reduzidos em $70 \%$ se os serviços de saúde seguissem os protocolos de tratamento preconizados 24. Em outro estudo com pacientes diabéticos, em Ribeirão Preto, São Paulo, foi demonstrado que a utilização desses protocolos aumentou a cobertura medicamentosa, a adesão ao plano alimentar e a atividade física, propiciando maior sucesso no tratamento desses pacientes 25 .

Os resultados obtidos em Belo Horizonte ressaltam a necessidade de implementar protocolos de tratamento para hipertensão e diabetes nas instituições de saúde e sensibilizar os prescritores para o seu uso, com vistas a melhorar a qualidade do atendimento, favorecendo a efetividade e a racionalidade terapêutica.

\section{Colaboradores}

M. F. Gontijo planejou o estudo, realizou o processamento e a análise dos dados e foi a redatora principal do artigo. A. Q. Ribeiro e F. A. Acurcio participaram da coleta de dados e colaboraram no planejamento do estudo, na análise dos dados e na redação do artigo. C. H. Klein e S. Rozenfeld colaboraram na análise dos dados e na redação final do artigo.

\section{Agradecimentos}

À Fundação Nacional de Saúde, ao Ministério da Saúde pelo financiamento do projeto. Ao apoio do Conselho Nacional de Desenvolvimento Científico e Tecnológico (CNPq; processo 306446/2003-0) e das Faculdades de Farmácia e de Medicina da Universidade Federal de Minas Gerais. 


\section{Referências}

1. Mosegui GBG, Rozenfeld S, Veras RP, Vianna CMM. Avaliação da qualidade do uso de medicamentos em idosos. Rev Saúde Pública 1999; 33:437-44.

2. Lima-Costa MF, Matos DL, Camargos VP, Macinko J. Tendências em dez anos das condições de saúde de idosos brasileiros: evidências da Pesquisa Nacional por Amostra de Domicílios (1998, 2003, 2008). Ciênc Saúde Coletiva 2011; 16:3689-96.

3. Sociedade Brasileira de Cardiologia/Sociedade Brasileira de Hipertensão/Sociedade Brasileira de Nefrologia. VI Diretrizes Brasileiras de Hipertensão. Arq Bras Cardiol 2010; 95(1 Suppl 1):1-51.

4. Sociedade Brasileira de Diabetes. Diretrizes da Sociedade Brasileira de Diabetes. Rio de Janeiro: Sociedade Brasileira de Diabetes; 2009.

5. Silva TR, Feldman C, Limaiii MHA, Cuce MR, Domingues RZL. Controle de diabetes Mellitus e hipertensão arterial com grupos de intervenção educacional e terapêutica de seguimento ambulatorial de uma Unidade Básica de Saúde. Saúde Soc 2006; 15:180-9.

6. Francisco PMSB, Belon AP, Barros MBA, Carandina L, Alves MCGP, Goldbaum M, et al. Diabetes autorreferido em idosos: prevalência, fatores associados e práticas de controle. Cad Saúde Pública 2010; 26:175-84.

7. Ribeiro AQ, Acurcio FA, Wick JY. Pharmacoepidemiology of the elderly in Brazil: state of the art. Consult Pharm 2009; 24:30-6.

8. Acurcio FA, Rozenfeld S, Ribeiro AQ, Klein CH, Moura CS, Andrade CR. Utilização de medicamentos por aposentados brasileiros. 1 - Metodologia e resultados de cobertura de inquérito multicêntrico. Cad Saúde Pública 2006; 22:87-96.

9. Dicionário de Especialidades Farmacêuticas DEF. 29a Ed. Rio de Janeiro: Editora de Publicações Científicas; 2000.

10. World Health Organization. Collaborating Centre for drug Statistics Metodhology. Anatomical Therapeutic Chemical (ATC) classification index with Defined Daily Doses (DDD's). Oslo: WHO Collaborating Centre for drug Statistics Metodhology; 2000.

11. Fick DM, Cooper JW, Wadw WE, Waller JL, Maclean JR, Beers MH. Updating the Beers criteria for potentially inapropriate medication use in older adults. Arch Intern Med 2003; 163:2716-24.

12. Schmidt MI, Duncan BB, Hoffmann JF, Moura L, Malta DC, Carvalho RMSV. Prevalência de diabetes e hipertensão no Brasil baseada em inquérito de morbidade autorreferida, Brasil, 2006. Rev Saúde Pública 2009; 43:74-82.

13. Lima-Costa MF, Peixoto SV, Firmo JOA. Validade da hipertensão arterial autorreferida e seus determinantes (Projeto Bambuí). Rev Saúde Pública 2004; 38:637-42.
14. Secoli SR, Figueras A, Lebrão ML, Lima FD, Santos JL. Risk of potential drug-drug interactions among Brazilian elderly: a population-based, cross-sectional study. Drugs Aging 2010; 27:759-70.

15. Ribeiro AQ, Rozenfeld S, Klein CH, César CC, Acurcio FA. Inquérito sobre uso de medicamentos por idosos aposentados, Belo Horizonte, MG. Rev Saúde Pública 2008; 42:724-32.

16. Rozenfeld S, Fonseca MJM, Acurcio FA. Drug utilization and polypharmacy among the elderly: a survey in Rio de Janeiro city, Brasil. Rev Saúde Pública 2008; 23:34-43.

17. Ministério da Saúde. Formulário terapêutico nacional 2010/Rename 2010. 2a Ed. Brasília: Ministério da Saúde; 2010.

18. Gus M, Fuchs FD. Análise crítica das evidências sobre o tratamento da hipertensão arterial no paciente com diabetes melito tipo 2. Rev Bras Hipertens 2006; 13:193-7.

19. Perroti TC, Campos Filho J, Uehara CA, Almada Filho CM, Miranda RD. Tratamento farmacológico da hipertensão no idoso. Rev Bras Hipertens 2007; 14:37-41.

20. Fuchs FD. Diuretics: still essential drugs for the management of hypertension. Expert Rev Cardiovasc Ther 2009; 7:591-8.

21. Strippoli GFM, Bonifati C, Craig M, Navaneethan SD, Craig JC. Inhibidores de la enzima convertidora de angiotensina y antagonistas de los receptores de angiotensina II para prevenir la progresión de la nefropatía diabética (Cochrane Review). Oxford: Update Software; 2008.

22. US Departament of Health and Human Services/ National Institute of Health/National Heart, Lung and Blood Institute. The seventh report of the Joint National Commitee on prevention, detection, evaluation, and treatment of high blood pressure. Bethesda: National Heart, Lung and Blood Institute; 2004.

23. Schroeter G, Trombeta T, Faggiani F, Goulart PV, Creutzberg M, Viegas K, et al. Terapia anti-hipertensiva utilizada por pacientes idosos de Porto Alegre/RS, Brasil. Sci Méd 2007; 17:14-9.

24. Melo DO, Ribeiro E, Storpirtis S. A importância dos estudos de utilização de medicamentos. Revista Brasileira de Ciências Farmacêuticas 2006; 42:475-85.

25. Zanetti ML, Otero LM, Peres DS, Santos MA, Guimarães FPM, Freitas MCF. Evolução do tratamento de pacientes diabéticos utilizando o protocolo Staged Diabetes Management. Acta Paul Enferm 2007; 20:338-44.

Recebido em 28/Set/2010

Versão final reapresentada em 10/Fev/2012 Aprovado em 28/Fev/2012 